\title{
Expression of the plasma prekallikrein gene: utilization of multiple transcription start sites and alternative promoter regions
}

\author{
Peter Neth', Marianne Arnhold', Viktoryia \\ Sidarovich1, Kanti D. Bhoola² and Edwin Fink ${ }^{1, *}$ \\ ${ }^{1}$ Abteilung für Klinische Chemie und Klinische \\ Biochemie, Chirurgische Klinik Innenstadt, Ludwig- \\ Maximilians-Universität München, Nussbaumstr. 20, \\ D-80336 München, Germany \\ ${ }^{2}$ Centre for Asthma, Allergy and Respiratory Research, \\ School of Medicine and Pharmacology, The University \\ of Western Australia, Sir Charles Gairdner Hospital, \\ Hospital Avenue, Nedlands WA 6009, Perth, Australia \\ ${ }^{*}$ Corresponding author \\ e-mail: fink@clinbio.med.uni-muenchen.de
}

\begin{abstract}
The plasma prekallikrein gene is expressed in many different human tissues at distinctly different levels and therefore tissue-specific control of the gene transcription is likely. In this study we demonstrate that transcription of the plasma prekallikrein gene can be initiated at multiple sites, for which at least four different promoters are utilized. A comparison of the genomic and mRNA sequences of mouse plasma prekallikrein revealed that the sequence segment that was formerly regarded as the first exon of the mouse plasma prekallikrein gene consists of three exons, with the first exon localized $14.2 \mathrm{kbp}$ upstream of the translation start. For the rat and human plasma prekallikrein genes, in silico analysis suggested an analogous exon-intron organization. Determination of the transcription start sites showed that in both mouse and human, the proximal and distal regions could be utilized for transcription initiation; however, the proximal region is preferred. A deletion mutation analysis of the proximal promoter region using a 1.7-kbp segment revealed a strong activating region immediately upstream of the known mRNA, followed by both a modest repressor and an enhancer region.
\end{abstract}

Keywords: alternative promoter; alternative transcription start site; Inr element; plasma kallikrein; plasma prekallikrein; promoter activity; TATA box; transcription start site; 5'-untranslated region heterogeneity.

\section{Introduction}

Plasma prekallikrein (PPK), the zymogen of the serine protease plasma kallikrein, is synthesized as a singlechain glycoprotein in hepatocytes and secreted into the bloodstream (Bhoola et al., 1992) where it circulates mainly as a complex with high-molecular-weight kininogen (HMWK). In vitro, conversion of plasma prekallikrein to active plasma kallikrein is achieved by cleavage of the peptide bond Arg371-Ile372 either in a fluid phase by a factor XII fragment or on negatively charged surfaces by activated factor XII (factor XIla) with HMWK as the cofactor (contact activation). Factor XIla-independent PPK activation has been shown to occur on the surface of endothelial cells by a protease (Colman and Schmaier, 1997; Motta et al., 1998, 2001) which has been identified by Shariat-Madar et al. (2002) as prolylcarboxypeptidase. In addition, it has been observed that activation of prekallikrein complexed to HMWK on endothelial cell surfaces may also be catalyzed by heat shock protein 90 (Joseph et al., 2002).

Biological activities attributed to PPK circulating in the blood are: involvement in the activation of neutrophils (Schapira et al., 1982, 1983; Wachtfogel et al., 1983); activation of the $\mathrm{C} 3$ convertase of the alternative pathway (DiScipio, 1982); induction of the fibrinolytic cascade by converting the pro-urokinase plasminogen activator to an active molecule (Ichinose et al., 1986; Hauert et al., 1989); and the release from HMWK of bradykinin, which regulates cellular events through specific receptor-coupling of second messengers (Blais et al., 2000; Blaukat, 2003). The formerly accepted role of PPK, together with factor XII and HMWK, in the coagulation pathway is no longer sustainable, since deficiencies of these proteins in plasma do not result in bleeding disorders (Colman and Schmaier, 1997; Davie, 2003).

Recently we demonstrated that the PPK gene is transcribed not only in the liver, but also at distinctly different levels in non-hepatic human tissues (Neth et al., 2001). Furthermore, in immunocytochemical studies plasma kallikrein/PPK has been specifically visualized in several human tissues, such as pancreas, kidney, testis and stomach (unpublished data). Since PPK in the blood originates essentially or totally from the liver (Colman and Wong, 1979), we concluded that the extrahepatically synthesized PPK has special functions at or near the site of its synthesis.

Interestingly, novel biological roles of plasma kallikrein that fit into the concept of local PPK functions have been reported recently. Hecquet et al. (2002) demonstrated that plasma kallikrein, as well as some other proteases, activates bradykinin B2 receptor directly, independent of kinin release. Selvarajan et al. (2001) recently reported that plasma kallikrein is required for adipogenesis. The enzyme mediates activation of a plasminogen cascade, which promotes adipocyte differentiation by degrading the fibronectin-rich extracellular matrix of preadipocytes. Peek et al. (2002) found that plasma kallikrein, as well as coagulation factor $\mathrm{XI}$, can activate pro-hepatocyte growth factor. Hepatocyte growth factor (HGF) is the 
Table 1 Alignment of mouse mRNA to mouse, rat and human genomic sequences.

\begin{tabular}{|c|c|c|c|c|c|}
\hline Exon and intron numbering & E1a & I1a & $\mathrm{E} 1 \mathrm{~b}$ & $11 \mathrm{~b}$ & E1c \\
\hline Length of newly identified mouse exons and introns & 87 & 1271 & 226 & 11852 & 291 \\
\hline Segments of mouse exons that map to rat genomic sequence & $3-87$ & & $1-226$ & & $2-290$ \\
\hline Length of mapping rat segments & 85 & & 228 & & 290 \\
\hline Identity, \% & 94 & & 92 & & 85 \\
\hline Gap, \% & 0 & & 1 & & 1 \\
\hline Length of rat intervening sequences & & 1271 & & 16400 & \\
\hline Segments of mouse exons that map to human genomic sequence & $6-85$ & & $1-175$ & & $80-258$ \\
\hline Length of mapping human segments & 80 & & 175 & & 188 \\
\hline Identity, \% & 81 & & 85 & & 74 \\
\hline Gap, \% & 2 & & 0 & & 9 \\
\hline Length of human intervening sequences & & 1198 & & 16734 & \\
\hline
\end{tabular}

Mouse exons $\mathrm{mE} 1 \mathrm{a}, \mathrm{mE} 1 \mathrm{~b}$ and $\mathrm{mE} 1 \mathrm{c}$ were identified by mapping mouse PPK mRNA to the mouse genomic sequence. The analogous rat and human exons E1a, E1b and E1c result from BLAST alignment of mouse PPK mRNA to rat and human genomic sequences (see the materials and methods section).

ligand for the tyrosine kinase receptor c-Met, and thus plasma kallikrein may also regulate processes that involve the HGF/c-Met signaling pathway. Akita et al. (2002) investigated the molecular mechanism underlying impaired liver regeneration by circulating endotoxin after partial hepatectomy and provided convincing evidence that this impairment is caused by plasma kallikrein-mediated activation of latent transforming growth factor $\beta$ (TGF- $\beta$ ). Interestingly, we recently found co-localization of PPK and TGF- $\beta 1$ in the tubules and mesangial cells of normal renal parenchyma and the tissue adjacent to clear cell carcinoma of the human kidney (unpublished data).

Synthesis in multiple tissues and involvement in various physiological processes suggest that a distinct regulation of PPK synthesis in particular tissues and cells is required, and we set out to characterize the transcriptional control of human PPK gene expression. In the current study, we show that the transcription of human PPK

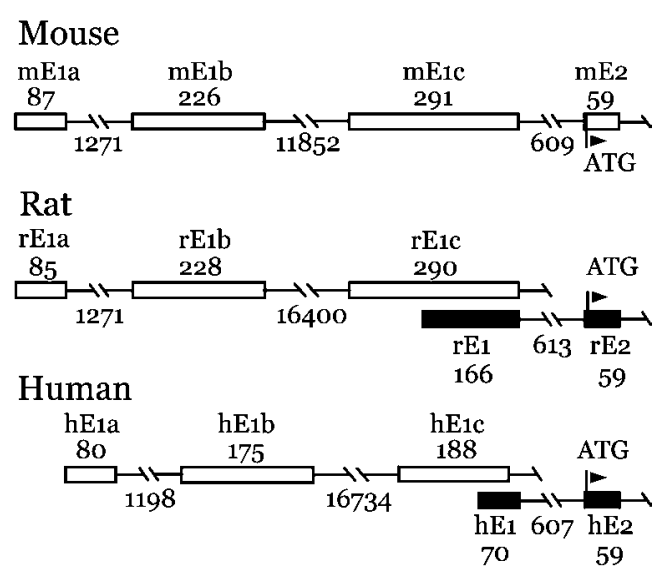

Figure 1 Organization of the $5^{\prime}$-regions of mouse, rat and human PPK genes.

The mouse exon-intron structure was obtained by mapping mouse PPK mRNA to the mouse genomic sequence. The putative exons E1a, E1b, and E1c (open boxes) for rat and human were assigned by aligning mouse mRNA to rat and human genomic sequences. The known human and rat exons 1 and 2 are shown as filled boxes; the start codons located at position 2 of exons $\mathrm{mE} 2$, rE2 and hE2 are indicated. gene can be initiated at multiple sites, which are up to $23 \mathrm{kbp}$ apart, indicating that different promoters can be utilized, presumably in a tissue- and/or cell type-specific manner. Alternatively spliced mRNA variants were detected, some of them indicating the existence of $\mathrm{N}$-terminally truncated PPK molecules. The major transcription start sites are close to the $5^{\prime}$-end of the known PPK mRNA (Chung et al., 1986). Examination by deletion analysis of the 1.7-kbp region upstream of the PPK mRNA for promoter activity showed that it contains both transcription-enhancing and -repressing segments.

\section{Results}

\section{Comparison of the mRNA and genomic sequences of human, mouse and rat PPK}

A comparison of the known PPK mRNAs from mouse (Seidah et al., 1990; NM_008455), rat (Seidah et al., 1989; Beaubien et al., 1991; NM_012725) and human (Chung et al., 1986; NM_000892) showed that the three 5 '-UTRs vary significantly in length, encompassing 605 , 167 and $71 \mathrm{bp}$, respectively. Therefore, the question arose as to whether the known human mRNA represents a full-length transcript of the PPK gene, and hence we examined this aspect by in silico analysis.

Mapping of the mRNAs to their respective genomic sequences confirmed for human (Yu et al., 2000) and rat (Beaubien et al., 1991) that the PPK genes consist of 15 exons with the coding sequence starting at position 2 of exon 2. In contrast, for mouse our alignment revealed that what was previously regarded as mouse exon 1 consists of three exons of 87, 226 and $291 \mathrm{bp}$ (tentatively designated $\mathrm{mE} 1 \mathrm{a}, \mathrm{mE} 1 \mathrm{~b}$ and $\mathrm{mE} 1 \mathrm{c})$, which are separated by introns I1a and I1b of 1271 and 11852 bp, respectively (Table 1, Figure 1).

The finding that mouse PPK mRNA contains two additional exons led to the view that analogous transcripts may also be formed in human and rat. In order to examine this possibility, we aligned the mouse exons $\mathrm{mE1a}$, $\mathrm{mE} 1 \mathrm{~b}$ and $\mathrm{mE} 1 \mathrm{c}$ to human chromosome 4 and rat chromosome 16, where the PPK genes are localized. As 
shown in Table 1 and Figure 1, nearly the complete exons $\mathrm{mE1a}, \mathrm{mE} 1 \mathrm{~b}$ and $\mathrm{mE1c}$ mapped to the rat genomic sequence with identities between $85 \%$ and $94 \%$. In analogy to the new mouse exons, these segments were provisionally designated $\mathrm{rE1a}, \mathrm{rE1b}$ and $\mathrm{rE1c}$. The intervening sequences $\mathrm{rl} 1 \mathrm{a}$ and $\mathrm{rl} 1 \mathrm{~b}$ were 1271 and $16400 \mathrm{bp}$ in length, respectively.

By alignment of the three mouse exons to the human genomic sequence, three segments of high similarity were also found (designated hE1a, hE1b, hE1c; Table 1, Figure 1), whereby $\mathrm{hE1c}$ did not overlap the complete conventional human exon 1 , but only 48 bp of its $5^{\prime}$-end. The lengths of the intervening sequences corresponding to mouse introns $1 \mathrm{a}$ and $1 \mathrm{~b}$ (1271 and $11852 \mathrm{bp}$ ) were 1198 and $16734 \mathrm{bp}$, respectively.

Taken together, comparison of the mRNA sequences of human, mouse and rat, and the alignment of the newly identified first three mouse exons to the rat and human genomic sequences suggested that the known rat and human mRNA sequences do not represent full-length transcripts of the PPK gene. Transcripts of the PPK gene with $5^{\prime}$-extensions (as compared to the known mRNAs) containing two additional exons might also exist in these two species. Conversely, it seemed possible that transcription of the mouse PPK gene can also be initiated within exon $\mathrm{mE1c}$ at start sites analogous to those of rat or human.

\section{The known human PPK mRNA is a full-length transcript and the transcriptional start sites in human liver, pancreas and kidney are diverse}

With the aim of characterizing the promoter of the human plasma prekallikrein gene, we determined first whether the known mRNA sequence (Chung et al., 1986) represented a full-length transcript and whether the same transcription starts were utilized in different tissues. To answer this question, transcription initiation sites were determined by RNA ligase-mediated rapid amplification of $5^{\prime}$-cDNA ends (RLM-RACE) with the PPK-specific primers positioned in exon 2 using poly $(A)^{+}$mRNA from human liver, pancreas and kidney, the three tissues with the highest PPK mRNA expression. The relative expression levels in liver, pancreas and kidney are 100\%, 68\% and $25 \%$, respectively (Neth et al., 2001).

By analysis of 31 clones from the three tissues, 15 different sites for transcription initiation were detected (Table 2); the distance on the genomic sequence between the most upstream and most downstream transcription start sites was $811 \mathrm{bp}$. Transcription start sites at or near position +1 of the known mRNA were detected in liver, pancreas and kidney, proving that the known human PPK mRNA represents a full-length transcript. Additional transcription start sites, further upstream or downstream of position +1 , were found exclusively in the kidney (Table 2): two transcripts had 5 '-extensions of 36 and $127 \mathrm{bp}$, and six had initiation sites within intron 1 . Accordingly, the segment of intron 1 upstream of the respective transcription start sites, as well as the conventional exon 1, are included in the promoter region. In this type of transcript the conventional first exon is missing, even though the complete PPK protein is encoded. Most interestingly, three additional transcription start
Table 2 Transcription start sites in human liver, pancreas, kidney and HepG2 cells.

\begin{tabular}{|c|c|c|c|c|c|}
\hline \multicolumn{2}{|c|}{$\begin{array}{l}\text { Position of } \\
\text { TSS }\end{array}$} & \multirow[t]{2}{*}{ Liver } & \multirow[t]{2}{*}{ Pancreas } & \multirow{2}{*}{$\begin{array}{c}\text { Kidney } \\
1\end{array}$} & \multirow[t]{2}{*}{ HepG2 } \\
\hline UTR & -127 & & & & \\
\hline UTR & -36 & & & 1 & \\
\hline UTR & -11 & & & & 4 \\
\hline UTR & -5 & & & & 1 \\
\hline E1 & +1 & 5 & 3 & 2 & 1 \\
\hline $\mathrm{E} 1$ & +3 & 1 & & & \\
\hline E1 & +4 & 1 & & & \\
\hline E1 & +6 & 3 & 3 & 1 & \\
\hline 11 & 136 & & & 1 & \\
\hline 11 & 243 & & & 1 & \\
\hline 11 & 248 & & & 1 & \\
\hline 11 & 265 & & & 1 & \\
\hline 11 & 334 & & & 1 & \\
\hline 11 & 436 & & & 1 & \\
\hline E2 & +75 & & & 1 & \\
\hline E2 & +76 & & & 2 & \\
\hline E2 & +77 & & & 1 & \\
\hline
\end{tabular}

TSS, transcription start site; UTR, 5' untranslated region; E1, E2, conventional exons 1 and 2; 11 , intron 1 . The numbering of positions in the $5^{\prime}$-upstream region and in exons 1 and 2 is relative to the $5^{\prime}$-end of the known mRNA sequence; in intron 1 the numbering refers to the $5^{\prime}$-end of intron 1 . Numbers in columns 3-6 indicate the number of clones with the respective transcription start site.

sites detected in the kidney were located in exon 2 in positions $+75,+76$ and +77 (the numbering refers to the known mRNA sequence); hence, the promoter region includes the complete intron 1 (607 bp, NT_022792; or 608 bp, AF232734 and our own sequencing results) and the conventional exon 1 (70 bp). Furthermore, since the start sites are downstream of the start codon at +72 , translation has to start at an ATG codon further downstream, causing a truncation by at least 38 amino acids. As a result, a PPK molecule would be produced that would be lacking not only the signal peptide, but also at least 19 amino acids of the mature protein.

\section{The human PPK gene has transcription initiation sites analogous to mouse and vice versa}

To address the question as to whether transcription start sites analogous to mouse PPK can be used in human and vice versa, we carried out a series of $5^{\prime}$-RLM-RACE analyses with mRNA from human and mouse liver. Using human liver mRNA and the gene-specific primer located in hE1a (cf. Figure 2), we detected two types of transcripts (types I and II, cf. Figure 2) with the transcription start sites 18.5 and $22.5 \mathrm{kbp}$ upstream of the previously reported human PPK mRNA (Yu et al., 2000). Type I transcripts $(n=2)$ started at positions resulting in lengths of hE1a of 83 and 110 bp (the $3^{\prime}$-end of hE1a identified by the alignment with mouse exon 1, cf. Table 1, was corrected following the GT-AG rule). The type II transcript $(n=1)$ contained two additional exons upstream of hE1a. The first upstream exon, hE1a-1 (135 bp), was separated from hE1a by $93 \mathrm{bp}$, and the second upstream exon, hE1a-2 (285 bp), from hE1a-1 by a 3562-bp intron.

Using mouse liver mRNA and the gene-specific primer positioned in $\mathrm{mE1c}$, we identified five different transcrip- 


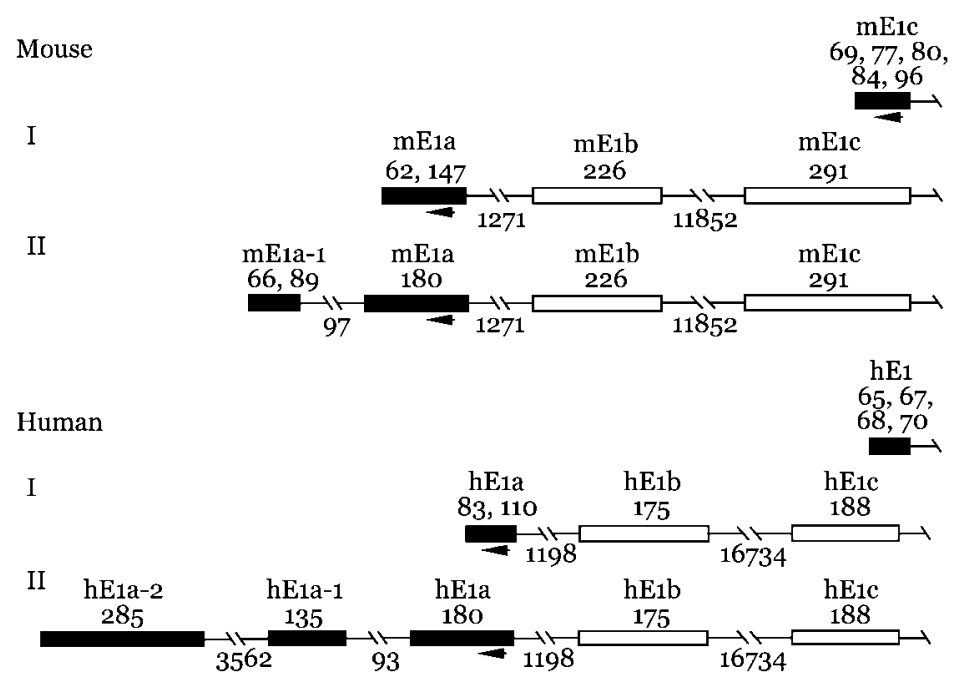

Figure 2 Determination of transcription initiation sites of the mouse and human PPK genes by RLM-RACE.

The positions of gene-specific primers (arrowheads) were located in mouse exons mE1c and mE1a, and in human exons hE2 (not shown) and hE1a. Numbers indicate the lengths of exons and introns in bp. Filled boxes represent the exons detected by RLMRACE and open boxes the exons identified by alignment of mouse mRNA to the genomic sequences. Roman numerals indicate the different types of transcripts (cf. results section).

tional start sites within $\mathrm{mE1c}$ (Figure 2). The resulting mE1c lengths of $69,77,80,84$ and 96 bp are similar in size to the conventional human exon 1. No transcripts corresponding to the known mouse mRNA (i.e., starting with $\mathrm{mE} 1 \mathrm{a}$ ) were detectable in this experiment. Therefore, we carried out a second 5'-RACE with the gene-specific primer in mE1a (Figure 2). We found two types of transcripts: type I $(n=2)$ started with $m E 1 a$, whereby the lengths of mE1a were 62 and $147 \mathrm{bp}$. Transcripts of type II $(n=2)$ had an additional upstream exon, mE1a-1, which was separated from mE1a (180 bp) by an intron of $97 \mathrm{bp}$. For mE1a-1, two transcription start sites were obtained, causing mE1a-1 lengths of 66 and $89 \mathrm{bp}$. No transcription start site representing exactly the $5^{\prime}$-end of the published mouse PPK mRNA (Seidah et al., 1990) was detected.

Additional evidence for the use of multiple transcription initiation sites was gained from a search of EST databases. For human, two sequences were found corresponding to our type II transcript (two additional exons upstream of hE1a; Figure 2), and two sequences starting at positions +3 and +9 of the known mRNA. For mouse, three sequences were detected with the $5^{\prime}$-ends in $\mathrm{mE1a}$, whereby none of the sequence start sites coincided exactly with that of the known mouse PPK mRNA. Furthermore, six sequences were found with one (our type II, cf. above) to three additional upstream exons. A total of 13 EST sequences had start sites within $\mathrm{mE1c}$; the resulting exon lengths would be $26,28,35,50,54,55$, $70,71,76,98$ and $119 \mathrm{bp}$, and thus would be similar to the size of the conventional human exon 1. A search of the Database of Transcriptional Start Sites (Suzuki et al., 2002) produced no results for human, whereas for mouse 10 transcription start sites within $\mathrm{mE} 1 \mathrm{c}$ are available, resulting in lengths of $\mathrm{mE} 1 \mathrm{c}$ of $26,50,54,55,70,76$ and $96 \mathrm{bp}$, but no distal transcription start sites are given.

Taken together, the RLM-RACE and the data mining results demonstrate that both in mouse and human, transcription of the PPK gene can be initiated at sites in regions that are distantly apart. Form the experimental results it can be concluded that transcription of the human PPK gene in the liver can be driven by three different promoters, namely the proximal promoter preceding position 1 of the conventional human exon $1, \mathrm{hE1}$, and two distal promoters located upstream of hE1a and hE1a-2, approximately 18.5 and $22.5 \mathrm{kbp}$ upstream of hE1 (Figure 2).

\section{Transcriptional start sites in HepG2 cells}

The human hepatoma cell line HepG2, which is widely used as a model system for hepatocytes, expresses PPK mRNA, as we had previously ascertained by RT-PCR (data not shown). Since we intended to use this cell line for promoter activity studies, we examined which transcription start sites are utilized in HepG2 cells. DNA sequence analysis of six PCR products obtained by RLM-RACE revealed that transcription of the PPK gene in HepG2 cells starts in the same region as in liver and pancreas (Table 2). The initiation sites identified represented positions $-11,-5$ and +1 . The similarity or identity of the start sites to those found in liver and pancreas indicates that in HepG2 cells the same promoter region is effective, which justified employing HepG2 cells as a model for studying the activity of the proximal PPK promoter.

\section{Deletion-mutation analysis of the proximal PPK promoter region}

A segment comprising 1675 bp of the $5^{\prime}$-flanking region and $22 \mathrm{bp}$ of the conventional exon 1 of human PPK gene was cloned by employing the 'Genome Walker' technique. The segment was linked to the 5 '-end of the promoterless secretory alkaline phosphatase reporter gene of the pSEAP2-Basic plasmid. This construct was transiently transfected into HepG2 cells and the promoter activity of the construct was determined by measuring 


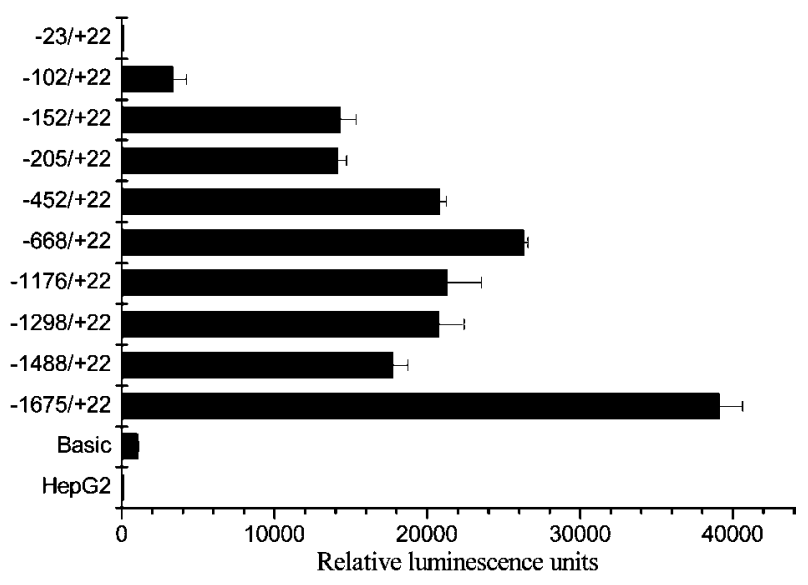

Figure 3 Deletion mutation analysis of the human PPK promoter region.

The $5^{\prime}$ - and $3^{\prime}$-ends of each deletion mutant are given on the left-hand side. Each construct was cloned into the PSEAP2 reporter gene plasmid and transiently transfected into HepG2 cells (in triplicate) and the supernatant was analyzed for alkaline phosphatase activity. Controls, indicated as Basic and HepG2, were HepG2 cells transfected with the reporter gene plasmid without insert and non-transfected HepG2 cells. The activity is given in relative luminescence units; the bars indicate the mean $\pm S D(n=3)$ of the SEAP activity.

the alkaline phosphatase activity released into the supernatant. The promoter activity of the $-1675 /+22$ construct was approximately 43-fold higher than of pSEAP2-Basic (Figure 3), indicating that the sequence encompasses a promoter region. Subsequent in silico analysis using MatInspector (Quandt et al., 1995) revealed that numerous consensus sequences for activating and repressing transcription factors are present within the $-1675 /+22$ segment. Taking into consideration the location of potential transcription factor binding sites, nine deletion variants of the full-length $-1675 /+22$ construct were prepared and examined for transcriptional activity. Figure 3 shows that the 10 reporter gene constructs displayed significantly different promoter activity. The highest activity was observed for the full-length construct $-1675 /+22$ (arbitrarily set to 100\%). Deletion of the sequence -1675 and -1488 resulted in decrease in activity to approximately $45 \%$. Subsequent deletion of -1488 to -1298 , -1298 to -1176 , and -1176 to -688 led to a gradual activity recovery to approximately $67 \%$. Further deletion of sequences -668 to -452 and -452 to -205 caused a decrease to $36 \%$; this activity remained unchanged by deletion to -152 . Removal of the next $50 \mathrm{bp}$ generated a sharp drop to $9 \%$ and deletion of the sequence -102/ -23 completely abolished this residual promoter activity.

\section{Discussion}

Experimental results and data mining for transcription start sites indicated that transcription of the PPK gene in human and mouse liver could be initiated in three different regions (Figure 2). However, neither in mouse nor in human were the distal initiation sites detected when $5^{\prime}$ RLM-RACE experiments were carried out with the genespecific primers located downstream of the proximal initiation sites, even though the distal start sites must in principle be detectable by such an experiment. However, the distal transcription start sites were detectable when the gene-specific primers in 5'-RLM-RACE experiments were located in the distal exons $\mathrm{mE1} 1 \mathrm{a}$ and $\mathrm{hE1} 1 \mathrm{a}$, respectively (Figure 2), namely, when experimental conditions selective for detecting the distal transcription start sites were chosen. This finding indicates that the proximal start sites represent the major region for transcription initiation. If transcription is mainly initiated near the proximal start site and the gene-specific primer located downstream to this site, then the number of formed short transcripts will be larger than the number of the long ones, which start at the distal transcription start sites. Thus, it would have been necessary in our experiments to screen a much larger number of clones than the 16 we examined to identify the long transcripts in addition to the short ones. Interestingly, the known mouse PPK mRNA described by Seidah et al. (1990) represents one of the long transcripts. Obviously, the authors were quite fortunate in finding one of these rare long transcripts by chance and against statistical expectancy. Taken together, our experiments elucidated that in both mouse and human liver transcription of the PPK gene can be initiated in at least three different regions, but the major transcription start region is the proximal one.

A comparison of the proximal transcription start sites in the human liver, pancreas and kidney, the three tissues with the highest PPK mRNA expression levels (Neth et al., 2001), unveiled tissue-dependent utilization of transcription starts (Table 2) and promoters. In the liver and pancreas, all start sites coincided with or were close to position +1 of the known PPK mRNA, whereas in the kidney additional start sites upstream and downstream of +1 were detected. For all the start sites downstream of position +1 the promoter differs substantially from the one upstream of +1 , as it includes part of intron 1 and exon 1 and can be regarded as alternative promoter(s). In all resulting transcripts the first exon will be missing. Moreover, those transcripts starting downstream of the start codon would give rise to a PPK molecule lacking both the signal peptide and at least 19 amino acids of the mature protein. Accordingly, because of the missing signal peptide, this molecule cannot be directed to the secretory pathway and therefore an intracellular role of the truncated PPK could be assumed. However, so far we have failed to demonstrate the existence of such a truncated PPK protein by Western blot experiments with human tissue extracts (data not shown).

Diverse transcription initiation is widespread throughout the genome, as revealed by Suzuki et al. (2001) who determined 5880 transcription start sites in 276 human genes in a $5^{\prime}$-full-length enriched cDNA library. In approximately $75 \%$ of these genes the most upstream and downstream transcription start sites were separated by up to $100 \mathrm{bp}$, and in 25\% the transcription starts were between 100 and $>200$ bp apart. Of the 276 genes, $15 \%$ had tightly clustered start sites and in only five genes did the transcription start site map to a single position. At present no analysis is available on whether different or single promoters drive gene transcription from multiple transcription start sites. However, it seems likely that a 


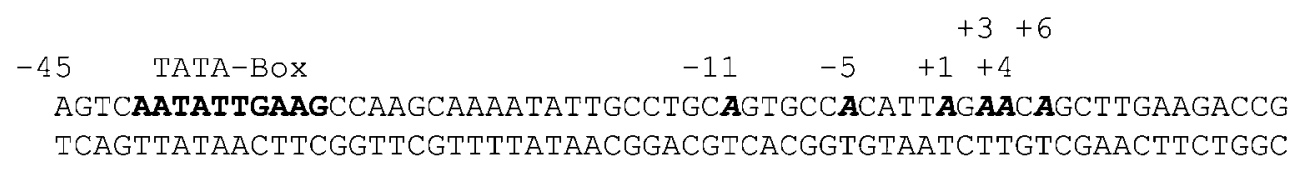

$-Y A N W-Y$

YYANWYY

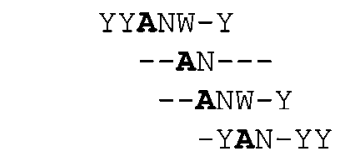

Inr-Elements

Figure 4 Transcription start sites of the PPK gene determined in human liver and pancreas.

A putative TATA box is shown in bold font. Experimentally determined transcription start sites are indicated by bold italics; the numbers are relative to the start site of the known mRNA. Consensus sequences of Inr elements are indicated below the sequence.

single promoter has the ability to drive transcription from different transcription start sites when they are located within an approximately 50-bp-wide region (Suzuki et al., 2001). Such use of a single promoter is certainly the case for the tightly clustered proximal transcription starts of the PPK gene.

The use of alternate promoters and multiple transcription start sites for a single gene has been described for multiple mammalian genes, and is regarded as part of the elaborate mechanisms that have evolved in multicellular organisms to provide a means of highly diversified temporal and spatial control of gene expression (Lemon and Tjian, 2000; Hochheimer and Tjian, 2003). In the case of $P P K$, the utilization of alternative promoters and/or alternative transcription start sites results in the expression of at least 20 different transcripts (Table 2 and Figure 2). This diversity of transcripts infers an intricate temporal and cell type-specific control of expression of the PPK gene and provides circumstantial evidence supporting our concept that PPK/plasma kallikrein plays hitherto unknown diverse functional roles in various tissues and cell types (Hermann et al., 1999; Neth et al., 2001). Supporting evidence for cell type-specific expression is also gained from our ongoing immunocytochemical studies (unpublished data), which for example show intense staining of PPK in distinct cells of human kidney tubules.

The experimental and data mining results demonstrate that, at least in the liver, it is the proximal region that is mainly used for transcription initiation. Therefore, for our studies on the regulation of expression of the human PPK gene, we chose to concentrate first on this major transcription start region. A TATA-box motive was identified at -41 to -32 (relative to the start of the known mRNA; Figure 4) which is a few bp upstream of the region most preferred for a TATA-box (Bucher, 1990), but can be expected to be a functional core promoter element. Consensus sequences of a second core promoter, the initiator element (Inr) (Smale, 1997), were identified for five of the six transcription start sites in liver, pancreas and HepG2 cells (Figure 4). No downstream promoter element (DPE) motive (G-A/T-C-G in the +30 region) (Burke and Kadonaga, 1997; Kutach and Kadonaga, 2000) was detectable. Taken together, the core promoter region of the PPK gene contains both a TATA-box and Inr motives in positions that can direct transcription initiation to any of the proximal transcription start sites found in liver, pan- creas and HepG2 cells, whereby the action of the motives may be paired or independent.

In our promoter activity study the high transcriptional activity of the $-1675 /+22$ reporter gene construct demonstrated that the 1.7-kbp sequence upstream of hE1 contains cis-acting elements important for the regulation of PPK gene transcription. Deletion mutation analyses (Figure 3) identified two regions with high stimulatory effects on transcription. The first one, the putative promoter, was found within the proximal sequence from $-668 \mathrm{bp}$ to the transcription start site, with the cis-acting elements mainly localized in the segments $-102 /-152$ and $-205 /-452$. The second one was detected in the 189-bp region -1488 to -1675 and is presumably an enhancer region. Transcription-repressing activity was found in the region -668 to -1488 between the promoter and the enhancer, mainly localized within the segments -668 to -1176 and -1298 to -1488 . In silico searches for transcription factor binding sites in the promoter region were carried out using two different methods (Quandt et al., 1995; Lenhard et al., 2003). However, even with the most stringent parameters, too many potential transcription factor binding sites were found to allow for a conclusive decision on which sites are biologically functional and which are 'innocent bystanders'. Therefore, further experiments have been designed to identify the critical binding sites.

PPK mRNA is expressed in many human tissues at different levels (Neth et al., 2001) and PPK participates as a protease in various biological processes. Therefore, in order to comply with the spatially and temporally varying demands for the functional activity of PPK, distinct mechanisms for fine-tuned transcriptional regulation of the PPK gene may be expected. In accordance, our experimental and data mining investigations show that at least four promoter regions and diverse transcription start sites can be utilized, and tissue-dependent alternative use of promoters and transcription starts is evident from a comparison of the start sites in liver and kidney.

\section{Materials and methods}

\section{Determination of transcriptional start sites of PPK}

Transcriptional start sites were determined by performing RNA ligase-mediated rapid amplification of 5'-CDNA ends (RLM- 
Table 3 Gene-specific primers for RLM-RACE.

\begin{tabular}{llll}
\hline Designation & & Position & \multicolumn{1}{c}{ Sequence $\left(5^{\prime}-3^{\prime}\right)$} \\
\hline $\begin{array}{l}\text { Human } \\
\text { hP-r139 }\end{array}$ & Exon 2 & $19560916-19560895$ & TAGCAAACAAGGAAATGAAATA \\
hP-r124-Nhe & Exon 2 & $19560901-19560876$ & GCTAGC-TGAAATAAGTTGCTTGCTTGAATAAA \\
hE1a-or71 & hE1a & $19541931-19541910$ & GCCAGCAGAGAAGGGCACGTAG \\
hE1a-ir47 & hE1a & $19541907-1941884$ & ATATGGATGGCGCCCTTGTGCATT \\
Mouse & & & \\
mP-or9146 & mE1a & $75-49$ & GAGGTCCAGCAGAAATGGCACATAGG \\
mP-ir9126 & mE1a & $55-29$ & ACATAGGCATAGGGATGGCGTCCTTGG \\
mP-or22799 & mE1c & $605-583$ & CCTGGAAGGATGGTCACGTTGTG \\
mP-ir22771 & mE1c & $577-550$ & GGAGGAGAGGGAGTCTTCACATGAAGAT \\
\hline
\end{tabular}

The position numbers refer to human NT_022792.15 and mouse PPK mRNA NM_008455.1. Underlined nucleotides indicate an Nhel recognition sequence.

RACE; Invitrogen, Karlsruhe, Germany) with $250 \mathrm{ng}$ of poly(A) ${ }^{+}$ mRNA from human liver, pancreas, and kidney and from mouse liver (Clontech, Heidelberg, Germany). 5'-RLM-RACE ensures that only full-length $5^{\prime}$-ends of mRNA molecules with an intact Cap structure are selectively reverse transcribed and amplified by nested PCR. For reverse transcription, random hexamer priming was employed. The gene-specific primers used for primary and nested PCR are listed in Table 3 . The PCR products were cloned into the plasmids PCRII-TOPO or PCR4-TOPO (Invitrogen) and the DNA sequences of the inserts were determined by a commercial service (MediGenomix, Martinsried, Germany).

\section{Isolation of the prekallikrein promoter region}

The upstream region of the human prekallikrein gene was cloned using the Genome Walker Kit (Clontech). Primary and nested PCRs were carried out with the Advantage Genomic Polymerase Mix (Clontech) using forward primers complementary to the adapter sequences and the gene-specific reverse primers $5^{\prime}$ AAT TGC TTC TTG GAG GTG AGT-3' and 5'-GCT AGC TGA ACG GTC TTC AAG CTG TTC T-3' (Nhel recognition site underlined) which correspond to nucleotides $60-40$ and 22-1 of the prekallikrein cDNA sequence. A 1.7-kbp product was obtained, which was cloned into the PCRII-TOPO vector and sequenced on both strands. The plasmid was cut with Mlul and Nhel and the resulting fragment was purified by agarose gel electrophoresis and cloned into the plasmid PSEAP2-Basic (Clontech), which was designated PPK/pSEAP2-Basic. Plasmid DNA was isolated from $100-\mathrm{ml}$ overnight cultures of single clones by the Mobius 1000 Kit (Novagen, Bad Soden, Germany) and stored at $-20^{\circ} \mathrm{C}$.

\section{Reporter plasmids}

Prekallikrein promoter segments of various lengths were generated by PCR with the PPK/pSEAP2 plasmid as a template. PCR was performed with Thermozyme (Invitrogen) using the primers shown in Table 4. The PCR products were cloned into the plasmid pCRII-TOPO and sequenced on both strands. Cloning into PSEAP-Basic and subsequent plasmid preparation was achieved as described above.

\section{Cell culture, transfection, and SEAP assay}

HepG2 cells were purchased from DSMZ (Braunschweig, Germany) and maintained at $37^{\circ} \mathrm{C}$ in RPMI medium (PAA, Pasching, Austria) supplemented with $10 \%$ fetal calf serum (FCS) (PAN Biotech, Aidenbach, Germany). Cells grown to confluence in 75$\mathrm{cm}^{2}$ flasks were harvested by trypsination (PAN Biotech) and replated in 12-well plates at approximately $40 \%$ confluence $(2 \mathrm{ml}$ of medium per well). The cells were grown overnight at $37^{\circ} \mathrm{C}$ in $5 \% \mathrm{CO}_{2}$ to $50-70 \%$ confluence. The medium was replaced by $0.9 \mathrm{ml}$ of fresh RPMI medium with $10 \% \mathrm{FCS}$, and $0.1 \mathrm{ml}$ of transfection mixture containing $3 \mu \mathrm{l}$ of FuGENE 6 transfection reagent (Roche, Mannheim, Germany) and $1 \mu \mathrm{g}$ of DNA in RPMI medium was added.

Secretory alkaline phosphatase (SEAP) activity was determined using the chemiluminescent SEAP assay system (Clontech). After 24 and/or $48 \mathrm{~h}, 15-\mu$ l aliquots from each cell culture supernatant were transferred into the wells of a 96-well microtest plate; $45 \mu$ l of dilution buffer was added and the plate was incubated for $30 \mathrm{~min}$ at $65^{\circ} \mathrm{C}$ to inactivate endogenous alkaline phosphatase. After cooling, $60 \mu \mathrm{l}$ of assay buffer and, after $5 \mathrm{~min}$ at room temperature, $60 \mu \mathrm{l}$ of CSPD substrate solution

Table 4 Primers used for the construction of reporter plasmids.

\begin{tabular}{lll}
\hline $\begin{array}{l}\text { Promoter } \\
\text { mutant }\end{array}$ & \multicolumn{1}{c}{ Position } & \multicolumn{1}{c}{ Nucleotide sequence $\left(5^{\prime}-3^{\prime}\right)$} \\
\hline$-23 /+22$ & $-23 /-2$ & ACGCGT-AATATTGCCTGCAGTGCCACAT \\
$-102 /+22$ & $-102 /-81$ & ACGCGT-GATGTTCATGGAATATGTTGAC \\
$-152 /+22$ & $-152 /-131$ & ACGCGT-GACATCACTCCCTGAAATAGTT \\
$-205 /+22$ & $-205 /-184$ & ACGCGT-GGGATGAGCGCTAGAAATCCT \\
$-452 /+22$ & $-452 /-431$ & ACGCGT-GTGGTGAAGTAGGTTCTATCT \\
$-668 /+22$ & $-668 /-647$ & ACGCGT-GCTAACGTGGAGGCTAGATAGA \\
$-1176 /+22$ & $-1176 /-1155$ & ACGCGT-AGTGTGGCCTTGCACTGAAGTA \\
$-1298 /+22$ & $-1298 /-1277$ & ACGCGT-TCTTAAGGCAATTATTTTAGC \\
$-1488 /+22$ & $-1488 /-1467$ & ACGCGT-CTAGTCTGCTGCTGAAGCTGTT \\
$-1675 /+22$ & $+22 /+1$ & GCTAGC-TGAACGGTCTTCAAGCTGTTCT \\
\hline
\end{tabular}

The numbers indicate the position relative to the $5^{\prime}$-end of the known mRNA. Underlined nucleotides represent Mlul and Nhel recognition sequences. 
(Clontech) were added. The luminescence was quantified after $10 \mathrm{~min}$.

\section{BLAST analyses and search for a TATA box}

For the alignment of sequences, the NCBI program BLAST 2 Sequences (Tatusova and Madden, 1999) was employed. The settings were: match, 1; mismatch, -2; gap open, 1 ; gap extension, 1; x_dropoff, 50; expect, 10; wordsize, 10; and filter, yes. Accession numbers of the PPK mRNA sequences are: human, NM_000892.2; mouse, NM_008455.1; and rat, NM_012725.1. Accession numbers of the genomic sequences are: human, NT_022792.15; mouse, NW_000342.1; and rat, NW_043042.1. For the TATA-box search, the program accessible at www.itb.cnr.it/webgene was used.

\section{Acknowledgments}

This work was supported in part by the Deutsche Forschungsgemeinschaft, grant Fl204/6-1.

\section{References}

Akita, K., Okuno, M., Enya, M., Imai, S., Moriwaki, H., Kawada, N., Suzuki, Y., and Kojima, S. (2002). Impaired liver regeneration in mice by lipopolysaccharide via TNF- $\alpha /$ kallikreinmediated activation of latent TGF- $\beta$. Gastroenterology 12 , 35-36.

Beaubien, G., Rosinski-Chupin, I., Mattei, M.G., Mbikay, M., Chrétien, M., and Seidah, N.G. (1991). Gene structure and chromosomal localization of plasma kallikrein. Biochemistry 30, 1628-1635.

Bhoola, K.D., Figueroa, C.D., and Worthy, K. (1992). Bioregulation of kinins: kallikreins, kininogens, and kininases. Pharmacol. Rev. 44, 1-80.

Blais, C., Marceau, F., Rouleau, J.L., and Adam, A. (2000). The kallikrein-kininogen-kinin system: lessons from the quantification of endogenous kinins. Peptides 21, 1903-1940.

Blaukat, A. (2003). Structure and signalling pathways of kinin receptors. Andrologia 35, 17-23.

Bucher, P. (1990). Weight matrix descriptions of four eukaryotic RNA polymerase II promoter elements derived from 502 unrelated promoter sequences. J. Mol. Biol. 212, 563-578.

Burke, T.W., and Kadonaga, J.T. (1997). The downstream core promoter element, DPE, is conserved from Drosophila to humans and is recognized by TAFII60 of Drosophila. Genes Dev. 11, 3020-3031.

Chung, D.W., Fujikawa, K., McMullen, B.A., and Davie, E.W. (1986). Human plasma prekallikrein, a zymogen to a serine protease that contains four tandem repeats. Biochemistry 25, 2410-2417.

Colman, R.W., and Schmaier, A.H. (1997). Contact system: a vascular biology modulator with anticoagulant, profibrinolytic, antiadhesive, and proinflammatory attributes. Blood 90, 3819-3843.

Colman, R.W., and Wong, P.Y. (1979). Kallikrein-kinin system in pathologic conditions. In: Handbook of Experimental Pharmacology, Vol. XXV (Supplement), E.G. Erdös, ed. (Berlin, Germany: Springer-Verlag), pp. 569-607.

Davie, E.W. (2003). A brief historical review of the waterfall/cascade of blood coagulation. J. Biol. Chem. 278, 50891-50832.

DiScipio, R.G. (1982). The activation of the alternative pathway C3 convertase by human plasma kallikrein. Immunology 45 , 587-595.

Hauert, J., Nicoloso, G., Schleuning, W.D., Bachmann, F., and Schapira, M. (1989). Plasminogen activators in dextran sulfate-activated euglobulin fractions: a molecular analysis of factor XII- and prekallikrein-dependent fibrinolysis. Blood 73, 994-999.

Hecquet, C., Becker, R.P., Tan, F.L., and Erdös, E.G. (2002). Kallikreins when activating bradykinin B-2 receptor induce its redistribution on plasma membrane. Int. Immunopharmacol. 2, 1795-1806.

Hermann, A., Arnhold, M., Kresse, H., Neth, P., and Fink, E. (1999). Expression of plasma prekallikrein mRNA in human nonhepatic tissues and cell lineages suggests special local functions of the enzyme. Biol. Chem. 380, 1097-1102.

Hochheimer, A., and Tjian, R. (2003). Diversified transcription initiation complexes expand promoter selectivity and tissuespecific gene expression. Genes Dev. 1, 130-132.

Ichinose, A., Fujikawa, K., and Suyama, T. (1986). The activation of pro-urokinase by plasma kallikrein and its inactivation by thrombin. J. Biol. Chem. 261, 3486-3489.

Joseph, K., Tholanikunnel, B.G., and Kaplan, A.P. (2002). Heat shock protein 90 catalyzes activation of the prekallikreinkininogen complex in the absence of factor XII. Proc. Natl. Acad. Sci. USA 99, 896-900.

Kutach, A.K., and Kadonaga, J.T. (2000). The downstream promoter element DPE appears to be as widely used as the TATA box in Drosophila core promoters. Mol. Cell. Biol. 20, 4754-4764.

Lemon, B., and Tjian, R. (2000). Orchestrated response: a symphony of transcription factors for gene control. Genes Dev. 10, 2551-2569.

Lenhard, B., Sandelin, A., Mendoza, L., Engström, P., Jareborg, N., and Wasserman, W.W. (2003). Identification of conserved regulatory elements by comparative genome analysis. J. Biol. 2, 13.

Motta, G., Rojkjaer, R., Hasan, A.A., Cines, D.B., and Schmaier, A.H. (1998). High molecular weight kininogen regulates prekallikrein assembly and activation on endothelial cells: a novel mechanism for contact activation. Blood 91, 516-528.

Motta, G., Shariat-Madar, Z., Mahdi, F., Sampaio, C.A.M., and Schmaier, A.H. (2001). Assembly of high molecular weight kininogen and activation of prekallikrein on cell matrix. Thromb. Haemost. 86, 840-847.

Neth, P., Arnhold, M., Nitschko, H., and Fink, E. (2001). The mRNAs of prekallikrein, factors $\mathrm{XI}$ and XII, and kininogen, components of the contact phase cascade are differentially expressed in multiple non-hepatic human tissues. Thromb. Haemost. 85, 1043-1047.

Peek, M., Moran, P., Mendoza, N., Wickramasinghe, D., and Kirchhofer, D. (2002). Unusual proteolytic activation of prohepatocyte growth factor by plasma kallikrein and coagulation factor Xla. J. Biol. Chem. 277, 47804-47809.

Quandt, K., Frech, K., Karas, H., Wingender, E., and Werner, T. (1995). MatInd and MatInspector: new fast and versatile tools for detection of consensus matches in nucleotide sequence data. Nucleic Acids Res. 23, 4878-4884.

Schapira, M., Despland, E., Scott, C.F., Boxer, L.A., and Colman, R.W. (1982). Purified human plasma kallikrein aggregates human blood neutrophils. J. Clin. Invest. 69, 1199-1202.

Schapira, M., Scott, C.F., Boxer, L.A., and Colman, R.W. (1983). Activation of human polymorphonuclear leukocytes by purified human plasma kallikrein. Adv. Exp. Med. Biol. 156, 747-753.

Seidah, N.G., Ladenheim, R., Mbikay, M., Hamelin, J., Lutfalla, G., Rougeon, F., Lazure, C., and Chretien, M. (1989). The cDNA structure of rat plasma kallikrein. DNA 8, 563-574.

Seidah, N.G., Sawyer, N., Hamelin, J., Mion, P., Beaubien, G., Brachpapa, L., Rochemont, J., Mbikay, M., and Chretien, M. (1990). Mouse plasma kallikrein. cDNA structure, enzyme characterization, and comparison of protein and mRNA levels among species. DNA Cell Biol. 9, 737-748.

Selvarajan, S., Lund, L.R., Takeuchi, T., Craik, C.S., and Werb, Z. (2001). A plasma kallikrein-dependent plasminogen cascade required for adipocyte differentiation. Nat. Cell Biol. 3, 267-275 
Shariat-Madar, Z., Mahdi, F., and Schmaier, A.H. (2002). Assembly and activation of the plasma kallikrein/kinin system: a new interpretation. Int. Immunopharmacol. 2, 1841-1849.

Smale, S.T. (1997). Transcription initiation from TATA-less promoters within eukaryotic protein-coding genes. Biochim. Biophys. Acta 1351, 73-88.

Suzuki, Y., Taira, H., Tsunoda, T., Mizushima-Sugano, J., Sese, J., Hata, H., Ota, T., Isogai, T., Tanaka, T., Morishita, S., et al. (2001). Diverse transcriptional initiation revealed by fine, large-scale mapping of mRNA start sites. EMBO Rep. 2, 388-393.

Suzuki, Y., Yamashita, R., Nakai, K., and Sugano, S. (2002). DBTSS: database of human transcriptional start sites and full-length cDNAs. Nucleic Acids Res. 30, 328-331.
Tatusova, T.A., and Madden, T.L. (1999). Blast 2 Sequences, a new tool for comparing protein and nucleotide sequences. FEMS Microbiol. Lett. 174, 247-250.

Wachtfogel, Y.T., Kucich, U., James, H.L., Scott, C.F., Schapira, M., Zimmerman, M., Cohen, A.B., and Colman, R.W. (1983). Human plasma kallikrein releases neutrophil elastase during blood coagulation. J. Clin. Invest. 72, 1672-1677.

Yu, H.R., Anderson, P.J., Freedman, B.I., Rich, S.S., and Bowden, D.W. (2000). Genomic structure of the human plasma prekallikrein gene, identification of allelic variants, and analysis in end-stage renal disease. Genomics 69, 225-234.

Received September 28, 2004; accepted December 6, 2004 